\title{
Analytical solutions of advection-dispersion equation for varying pulse type input point source in one-dimension
}

\author{
Dilip Kumar Jaiswal *, Atul Kumar \\ Department of Mathematics \& Astronomy, Lucknow Universityy, Lucknow, India-226007, INDIA \\ *Corresponding authors- email: dilip3jais@gmail.com (D. K. Jaiswal), atul_tusaar@rediffmail.com (A. Kumar)
}

\begin{abstract}
Analytical solutions are obtained for a one-dimensional advection-dispersion equation with variable coefficients in a longitudinal domain. Two cases are considered. In the first one the solute dispersion is time dependent along a uniform flow in a semi-infinite domain while in the second case the dispersion and the velocity both have spatially dependent expressions. Analytical solutions are obtained by introducing new independent variables with the help of certain transformations. Result and discussions are given by different graphs.
\end{abstract}

Keywords: Advection; Dispersion; Contaminants; Aquifer; Groundwater.

\section{Introduction}

The contaminants in aquifer systems migrate with ground water flow, any factors that may affect groundwater flow are also likely to influence the migration of contaminants in aquifers. Because contaminants are chemicals or bacteria or virus which are mostly physically, chemically and biologically active, the transport of contaminants are subject to physical, chemical and biological activities, such as contaminant density, adsorption and desorption, retardation, degradation and chemical-biological reactions. Analytical solutions, numerical simulations, and experiment and field observations are used to address groundwater flow and contaminant transport problems in aquifers.

Contaminant (solute) transport through a medium is described by a partial differential equation of parabolic type and it is usually known as advection-dispersion equation. Advection-dispersion equation is applicable in many disciplines like groundwater hydrology, chemical engineering bio sciences, environmental sciences and petroleum engineering to describe the behavior of solute concentration. In earlier, this equation along with a set of initial and boundary conditions has been solved for uniform dispersion and velocity. A list of previous investigators are Bastian and Lapidus (1956), Banks and Ali (1964), Ogata (1970) and Marino (1974), Al-Niami and Rushton (1977). Most of them take into account the effects of adsorption, first order decay, zero order production. Such solutions have been compiled by Lindstrom and Boersma (1989). Coming nearer to real problems, Banks and Jerasate (1962), Hunt (1978), Kumar (1983) considered the porous media flow unsteady/ non-uniform. Yates (1990) developed an analytical solution for describing the transport of dissolved substances in heterogeneous porous media with a distance dependent dispersion which may be used to characterize differences in the transport process relative to classical convection-dispersion equation for constant hydrodynamic dispersion in the porous medium. van Kooten (1996) has given a method to solve the transport equations for a kinetically adsorbing solute in a porous medium with spatially varying velocity field and dispersion coefficients.

Chen et al. (2003) presented a novel mathematical model to describe solute transport in a radially convergent flow field with scale-dependent dispersion. The scale-dependent advection-dispersion equation in cylindrical coordinates derived based on the dispersivity is assumed to increase linearly with the distance of the solute transported from its input source. The Laplace transformed power series technique is applied to solved the radially scale-dependent advection-dispersion equation with variable coefficients. Meerschaert and Tadjeran (2004) used fractional advection-dispersion equations in groundwater hydrology to model the transport of passive tracers carried by fluid flow in a porous medium. They developed practical numerical methods to solve one dimensional fractional advection-dispersion equations with variable coefficients on a finite domain. The practical application of 
these results is illustrated by modeling a radial flow problem. Use of the fractional derivative allows the model equations to capture the early arrival of tracer observed at a field site. Su et al. (2005) presented a specific form of the Fokker-Planck equation with a time- and scale-dependent dispersivity for modeling solute transport in saturated heterogeneous porous media. By taking a dispersivity in the form of separable power-law dependence on both time and scale, they show the existence of similarity solutions. Explicit closed-form solutions are then derived for an instantaneous point-source (Dirac delta function) input, and for constant concentration and constant flux boundary conditions on a semi-infinite domain. Moreira et al. (2006) reported the state-of-art of the ADMM (Advection Diffusion Multilayer Model) model, with solutions of the one and two-dimensional, steady state and time dependent advection-diffusion equation obtained by Laplace transform application. They also presented the novelty of solution of the advection-diffusion equation considering non-local effects in the turbulence closure and a critical evaluation of the influence of Gaussian quadrature points in the solutions. Sirin (2006) assumed pore flow velocity to be a non divergence-free, unsteady and non-stationary random function of space and time for ground water contaminant transport in a heterogeneous media.

Smedt (2006) presented analytical solutions for solute transport in rivers including the effects of transient storage and first order decay. Kartha and Srivastava (2008) studied one-dimensional numerical simulation is carried out of the effect of immobile water content on the time of appearance of contaminant at the bottom of an unsaturated column. Jaiswal et al. (2009) and Kumar et al. (2010) obtained analytical solutions for temporally and spatially dependent solute dispersion of point source input concentration in one dimensional semi-infinite media. Mohebbi and Dhghan (2010) proposed a high-order accurate method for solving the onedimensional heat and advection-diffusion equations in addition to high-order of accuracy of the proposed method. Numerical results show that the compact finite difference approximation of fourth-order and the cubic $C^{1}$ - spline collocation method give an efficient method for solving the one-dimensional heat and advection-diffusion equations.

Warrick et al. (1972) examined miscible displacement processes with time-varying velocity and dispersion coefficients and solutions are used in the analysis of experimental data, both for field infiltration with a slug of solute and for psychrometric measurements of salt fronts in a laboratory sand column. Zoppou and Knight (1997) presented one-dimensional analytical solutions for equation in which they considered solute dispersion varying with square of position variable while velocity varies with the position variable. In the present work two dispersion problems are addressed using Laplace Transform Technique, analytical solutions are obtained for one-dimensional advection diffusion equations describing the dispersion of pulse type input point source along temporally dependent and spatially dependent flow domains, respectively, through semi-infinite medium. The point source of varying nature is considered. The spatial dependence due to inhomogeneity of the medium is demonstrated by linearly interpolated velocity in position variable. Introduction of new space variables enable to reduce the advection-dispersion equation in both the problems into one-dimensional equation with constant coefficients.

\section{Analytical solutions}

In one space dimension the linear advection-dispersion equation may be written as

$$
\frac{\partial C}{\partial t}=\frac{\partial}{\partial x}\left[D(x, t) \frac{\partial C}{\partial x}-u(x, t) C\right]
$$

where $C$ is the solute concentration, $x$ is space variable, $t$ is time, $D(x, t)$ is solute dispersion and is called the dispersion coefficient if it is uniform and steady, and $u(x, t)$ is the velocity of the medium . It is derived on the principle of conservation of mass and Fick's laws of diffusion. If the medium is porous the velocity of flow satisfies the Darcy's law.

Let us write $D(x, t)=D_{0} f_{1}(x, t)$ and $u(x, t)=u_{0} f_{2}(x, t)$ in Eq. (1) as

$$
\frac{\partial C}{\partial t}=\frac{\partial}{\partial x}\left(D_{0} f_{1}(x, t) \frac{\partial C}{\partial x}-u_{0} f_{2}(x, t) C\right) \text {, }
$$

where $D_{0}$ and $u_{0}$ are constants.

Let us introduce a new independent variable, $X$ by following transformation (Jaiswal et al., 2009, Kumar et al., 2010)

$$
\frac{\partial X}{\partial x}=-\frac{1}{f_{1}(x, t)} \quad \text { or } \quad X=-\int \frac{d x}{f_{1}(x, t)}
$$

As a result Eq. (2) becomes

$$
f_{1}(x, t) \frac{\partial C}{\partial t}=D_{0} \frac{\partial^{2} C}{\partial X^{2}}+u_{0} \frac{\partial}{\partial X}\left(f_{2}(x, t) C\right)
$$

Two cases are considered. In the first case a time dependent dispersion along a uniform flow is considered while the second case deals with space dependent dispersion along a non-uniform flow in a semi-infinite domain. 


\subsection{Temporally dependent dispersion along uniform flow}

Let $f_{1}(x, t)=f(m t)$, and $f_{2}(x, t)=1$, where $m$ is a resistive coefficient whose dimension is inverse of that of the time variable $t$. $f(m t)$ is chosen such that for $m=0$ or $t=0, f(m t)=1$. Thus $f(m t)$ is an expression in the non-dimensional variable $m t$. Then from Eq. (3) we have

$$
X=-\frac{x}{f(m t)}
$$

Eq. (4) will become

$$
f(m t) \frac{\partial C}{\partial t}=D_{0} \frac{\partial^{2} C}{\partial X^{2}}+u_{0} \frac{\partial C}{\partial X}
$$

Let us introduce a new time variable using the following transformation (Crank, 1975)

$$
T=\int_{0}^{t} \frac{d t}{f(m t)}
$$

The partial differential equation (6) reduces into that with constant coefficients as

$$
\frac{\partial C}{\partial T}=D_{0} \frac{\partial^{2} C}{\partial X^{2}}+u_{0} \frac{\partial C}{\partial X}
$$

Let the domain is initially solute free. The input source concentration is not always uniform due to many reasons. For it, varying pulse type input source concentration is considered. An input concentration of varying nature is assumed at the origin of the domain. It is assumed to be $C_{0}$ until $t=t_{0}$ and beyond that it starts decrease instead of becoming zero. The second boundary condition is considered of solution type of homogeneous nature. Thus the initial and boundary conditions in mathematical form are as follows:

$$
\begin{aligned}
& C(x, t=0)=0, x \geq 0, \\
& -D(x, t) \frac{\partial C}{\partial x}+u(x, t) C(x, t)=\left\{\begin{array}{ll}
u_{0} C_{0} & 0<t \leq t_{0} \\
0 & , t>t_{0}
\end{array}, x=0\right. \\
& C(x, t)=0, x \rightarrow \infty, t \geq 0
\end{aligned}
$$

These conditions in terms of new space and time variable may be written as

$$
\begin{aligned}
& C(X, T)=0, \quad-\infty<X \leq 0, T=0, \\
& D_{0} \frac{\partial C}{\partial X}+u_{0} C(X, T)=\left\{\begin{array}{ll}
u_{0} C_{0} & 0<T \leq T_{0} \\
0 & T>T_{0}
\end{array} \quad X=0\right. \\
& C(X, T)=0 \quad, X \rightarrow-\infty, T \geq 0
\end{aligned}
$$

Using

$$
Z=-X \text {, }
$$

The initial and boundary value problem becomes

$$
\begin{aligned}
& \frac{\partial C}{\partial T}=D_{0} \frac{\partial^{2} C}{\partial Z^{2}}-u_{0} \frac{\partial C}{\partial Z} \\
& C(Z, T)=0, \quad Z \geq 0, \quad T=0, \\
& -D_{0} \frac{\partial C}{\partial Z}+u_{0} C=\left\{\begin{array}{ll}
u_{0} C_{0} & 0<T \leq T_{0} \\
0 & , T>T_{0}
\end{array} \quad Z=0\right. \\
& C(Z, T)=0 \quad, Z \rightarrow \infty, T \geq 0
\end{aligned}
$$

Now introducing a new dependent variable by following transformation

$$
C(Z, T)=K(Z, T) \exp \left[\frac{u_{0}}{2 D_{0}} Z-\frac{u_{0}^{2}}{4 D_{0}} T\right],
$$

The set of Eqs. (14) to (16) reduced into

$$
\begin{aligned}
& \frac{\partial K}{\partial T}=D_{0} \frac{\partial^{2} K}{\partial Z^{2}} \\
& K(Z, T)=0, T=0, Z \geq 0
\end{aligned}
$$




$$
\begin{aligned}
& -D_{0} \frac{\partial K}{\partial Z}+\frac{u_{0}}{2} K=\left\{\begin{array}{ll}
u_{0} C_{0} \exp \left(\alpha^{2} T\right) \\
0
\end{array}, \begin{array}{l}
0<T \leq T_{0} \\
T>T_{0}
\end{array}, Z=0 ; \quad \alpha^{2}=\frac{u_{0}^{2}}{4 D_{0}}\right. \\
& K(Z, T)=0, \quad Z \rightarrow \infty, T \geq 0
\end{aligned}
$$

Eqs. (18) and (20b) are satisfied by a solution (Crank, 1984)

$$
K(Z, T)=A \operatorname{erfc}\left(\frac{Z}{2 \sqrt{D_{0} T}}\right),
$$

where $A$ is an arbitrary constant and may be obtained by using (20a). Further reusing (17) the desired solution is

$$
\begin{aligned}
& C(Z, T)=F(Z, T), \quad 0<T \leq T_{0} \\
& C(Z, T)=F(Z, T)-F\left(Z, T-T_{0}\right), T>T_{0}
\end{aligned}
$$

where $\quad F(Z, T)=u_{0} C_{0} \frac{\exp \left(\frac{u_{0}}{2 D_{0}} Z\right)}{\left(\frac{u_{0}}{2}+\frac{\sqrt{D_{0}}}{\sqrt{\pi T}}\right)} \operatorname{erfc}\left(\frac{Z}{2 \sqrt{D_{0} T}}\right), Z=\frac{x}{f(m t)}$ and $T=\int_{t=0}^{t} \frac{d t}{f(m t)}$.

\subsection{Spatially dependent dispersion along non-uniform flow}

In this case let us consider following expressions for dispersion and velocity

$$
f_{1}(x, t)=(1+a x)^{2} \text {, and } f_{2}(x, t)=(1+a x) \text {, }
$$

where $a x$ is a non-dimensional and $a$ is non-zero real constant has the dimension of inverse of space variable accounting for the variations in velocity and dispersion due to inhomogeneity. To have these variations small, let $0<a x \leq 1$. Different values of $a$ will represent different media having different inhomogeneous nature. From the Eq. (3) we will have

$$
X=\frac{1}{a(1+a x)}
$$

Eq. (4) will become

$$
\frac{\partial C}{\partial t}=a^{2} D_{0} X^{2} \frac{\partial^{2} C}{\partial X^{2}}+a u_{0} X \frac{\partial C}{\partial X}-a u_{0} C
$$

Let us assume initial and boundary conditions same as of case first in a longitudinal semi-infinite domain and using Eq. (3) and the following transformation

$$
Z=-\log a X
$$

respectively. The initial and boundary value problem in terms of the new independent variables $(Z, T)$ may be written as

$$
\begin{aligned}
& \frac{\partial C}{\partial t}=a^{2} D_{0} \frac{\partial^{2} C}{\partial Z^{2}}-\left(a u_{0}-a^{2} D_{0}\right) \frac{\partial C}{\partial Z}-a u_{0} C \\
& C(Z, t)=0, \quad Z \geq 0, t=0, \\
& -a D_{0} \frac{\partial C}{\partial Z}+u_{0} C=\left\{\begin{array}{ll}
u_{0} C_{0} & 0<t \leq t_{0} \\
0 & t>t_{0}
\end{array}, Z=0\right. \\
& C(Z, t)=0, Z \rightarrow \infty, t \geq 0
\end{aligned}
$$

Further using a new dependent variable by transformation

$$
C(Z, t)=K(Z, t) \exp \left[\frac{w_{0}}{2 a D_{0}} Z-\left(\frac{w_{0}{ }^{2}}{4 a^{2} D_{0}}+a u_{0}\right) t\right], w_{0}=a u_{0}-a^{2} D_{0},
$$

the set of Eqs. (26) to (28) reduces into

$$
\begin{aligned}
& \frac{\partial K}{\partial t}=a^{2} D_{0} \frac{\partial^{2} K}{\partial Z^{2}} \\
& K(Z, t)=0, t=0, Z \geq 0
\end{aligned}
$$




$$
\begin{aligned}
& -\frac{\partial K}{\partial Z}+\frac{u_{0}+a D_{0}}{2 a D_{0}} K=\left\{\begin{array}{l}
\frac{u_{0} C_{0}}{a D_{0}} \exp \left\{\left(\frac{w_{0}{ }^{2}}{4 a^{2} D_{0}}+a u_{0}\right) t\right\} \\
0
\end{array}\right. \\
& K(Z, t)=0, Z \rightarrow \infty, t \geq 0
\end{aligned}
$$

Eqs. (30) and (32b) are satisfied by a solution (Crank, 1984)

$$
K(Z, t)=B \operatorname{erfc}\left(\frac{Z}{2 a \sqrt{D_{0} t}}\right),
$$

where $B$ is an arbitrary constant and may be eliminated from the general solution by using the condition (32a) and lastly using back transformation, one may get the desired solution as

$$
\begin{aligned}
& C(Z, t)=G(Z, t) \quad, 0<t \leq t_{0} \\
& C(Z, t)=G(Z, t)-G\left(Z, t-t_{0}\right), t>t_{0}
\end{aligned}
$$

where $G(Z, t)=\frac{u_{0} C_{0} \exp \left(\frac{w_{0}}{2 a^{2} D_{0}} Z\right)}{a D_{0}\left(\frac{u_{0}+a D_{0}}{2 a D_{0}}+\frac{1}{\sqrt{\pi D_{0} t}}\right)} \operatorname{erfc}\left(\frac{Z}{2 a \sqrt{D_{0} t}}\right), w_{0}=a u_{0}-a^{2} D_{0}, Z=\log \{(1+a x)\}$.

\section{Result and discussions}

The analytical solutions are illustrated with the help of set of input data to understand the concentration distribution behavior in the two cases, respectively. All the figures are drawn for varying pulse type input source concentration along a flow in a finite domain $0 \leq x(\mathrm{~km}) \leq 1$ of semi-infinite region. The set of input data for case-I i.e., for time dependent are $C_{0}=1.0$, $u_{0}=0.27(\mathrm{~km} / \mathrm{yr}), D_{0}=0.39\left(\mathrm{~km}^{2} / \mathrm{yr}\right)$ and $m=0.1(\mathrm{yr})^{-1}$. The time of elimination of the source concentration is considered $t_{0}=1.2(\mathrm{yr})$. The figures are drawn at $t(\mathrm{yr})=0.4,0.7$ and 1.0 for $t \leq t_{0}$ and at $t(\mathrm{yr})=1.3,1.6$ and 1.9 for $t>t_{0}$. Fig. 1a and $1 \mathrm{~b}$ depicts the distribution of concentration for $f(m t)=\exp (-m t)$ which is of decreasing nature, at different time for $t \leq t_{0}$ and $t>t_{0}$, respectively. From Fig. 1a, it is observed that the solute concentrations are increase with increasing time at initial stage in the presence of source.

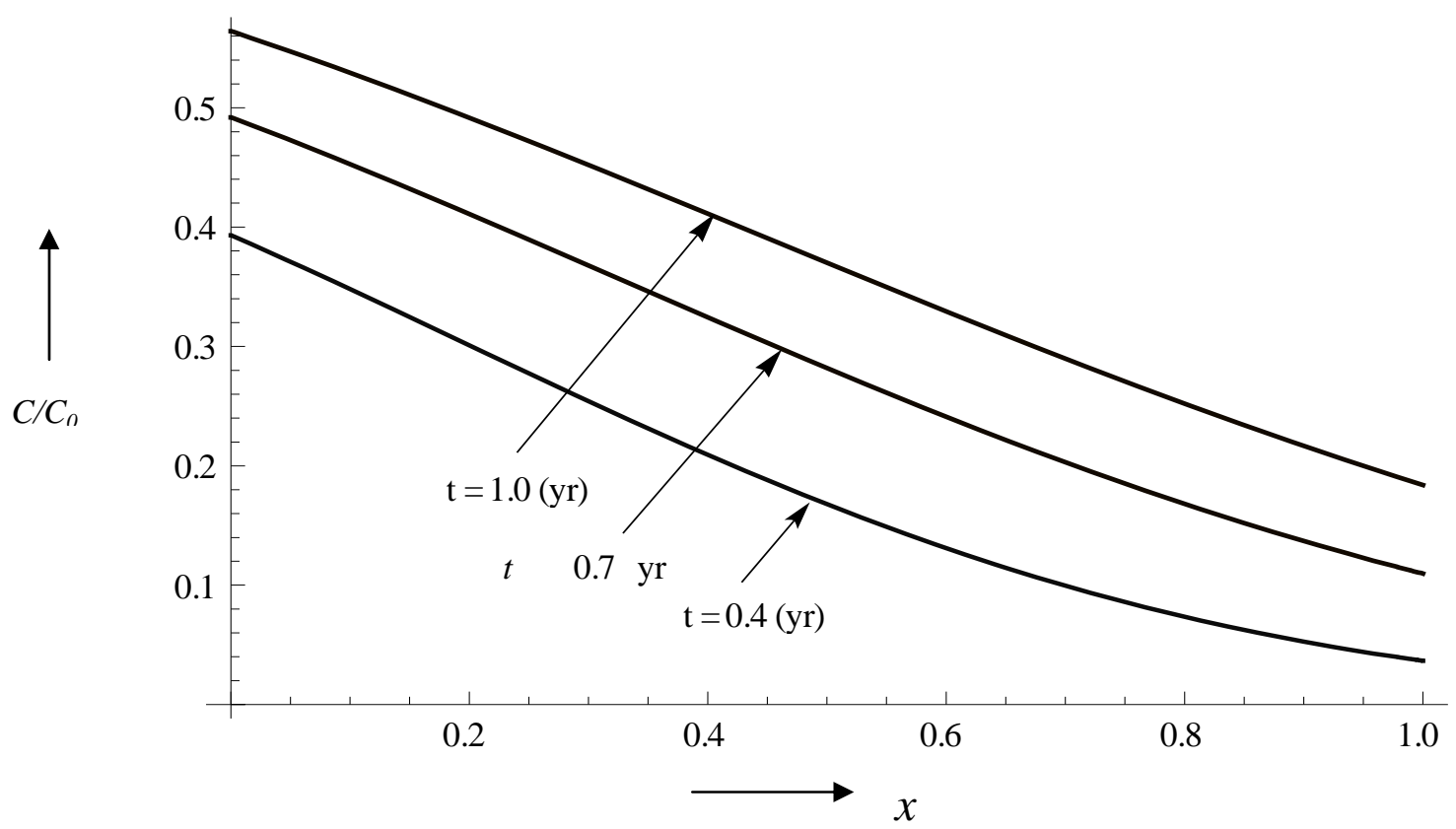

Figure 1a. Distribution behavior of solute in presence of source concentration for time dependent dispersion of decreasing function at different time. 


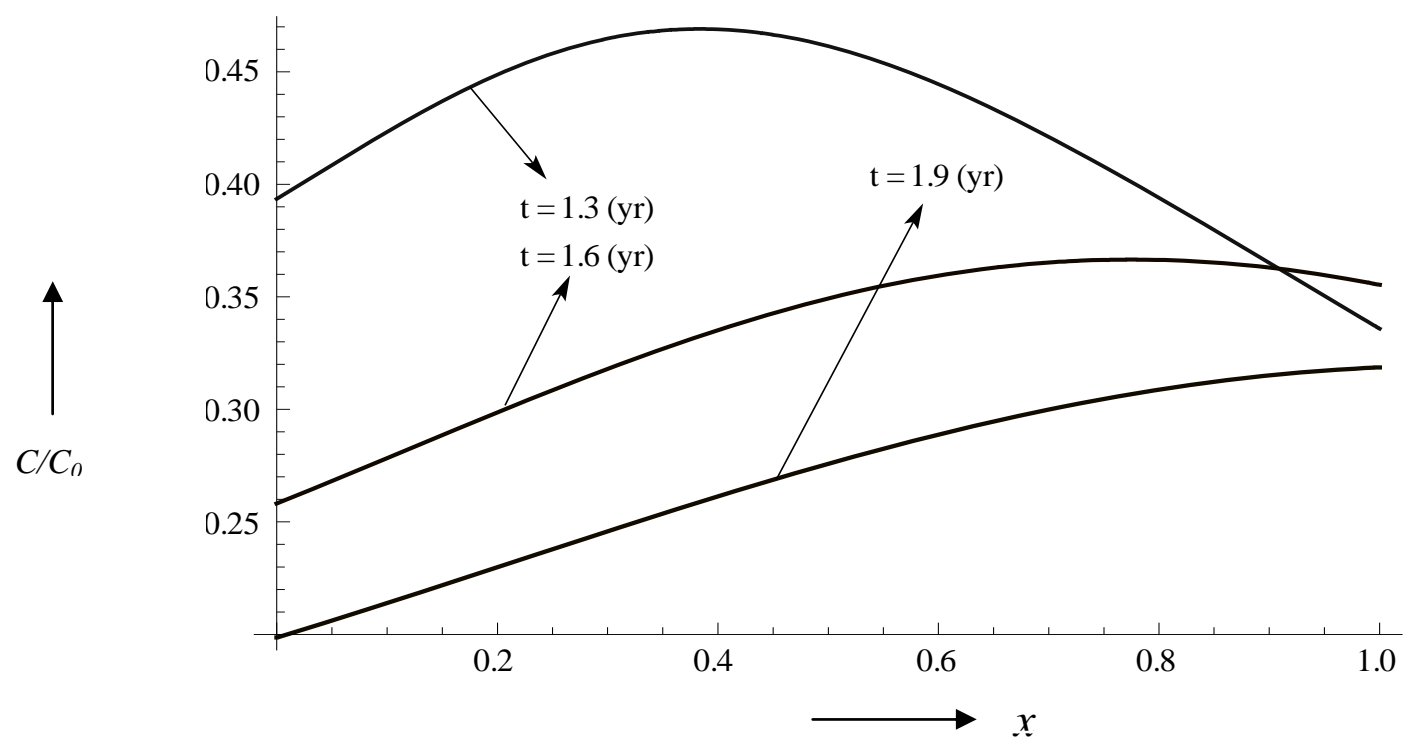

Figure 1b. Distribution behavior of solute in absence of source concentration for time dependent dispersion of decreasing function at different time.

In Fig. 1b, when source is absent i.e., absence of source the distribution of solute concentration achieve reverse trained. The trained of distribution of concentration is similar in all function of $f(m t)$.

Fig. $2 \mathrm{a}$ and $2 \mathrm{~b}$, shows the concentration values for case-II i.e., for spatially dependent dispersion and velocity for $t \leq t_{0}$ at time $t$ $(\mathrm{yr})=0.4,0.7,1.0$ and for $t>t_{0}$ at time $t(\mathrm{yr})=1.3,1.6,1.9$ respectively where $a=1.0(\mathrm{~km})^{-1}$. The input data for this case are $C_{0}=1.0, \quad u_{0}=0.21(\mathrm{~km} / \mathrm{yr})$ and $D_{0}=0.31\left(\mathrm{~km}^{2} / \mathrm{yr}\right)$. The source of elimination is considered same as in case first i.e., $t_{0}=1.2(\mathrm{yr})$.

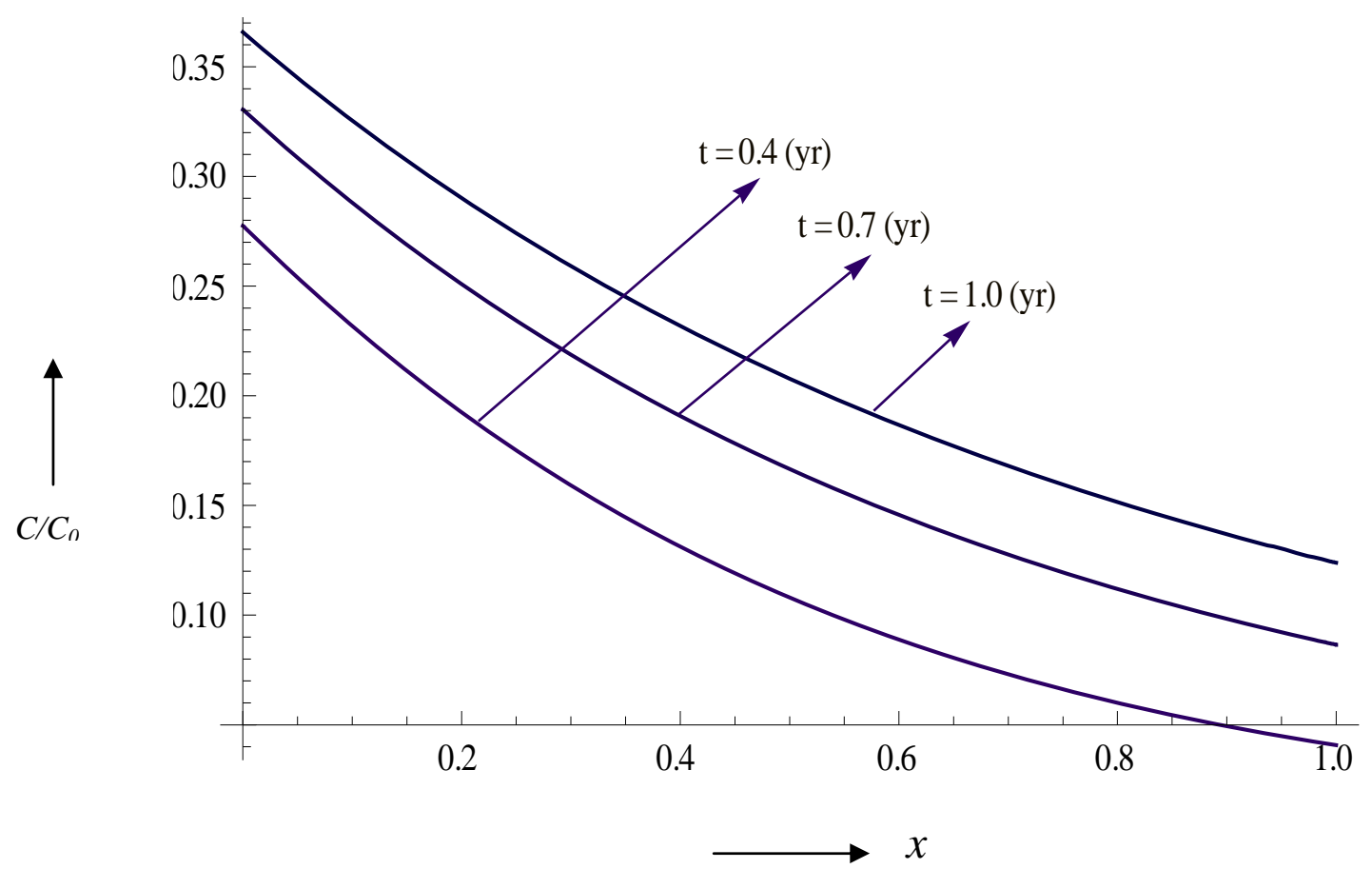

Figure 2a. Distribution behavior of solute in presence of source concentration for spatially dependent dispersion at different time. 
Nature of solute distribution of spatially dependent dispersion in Fig. 2a, in presence of source concentration at initial point is higher for higher time but dispersion is faster for lower time in comparison of higher time although, in Fig. 2b in absence source, dispersion of solute is faster for higher time.

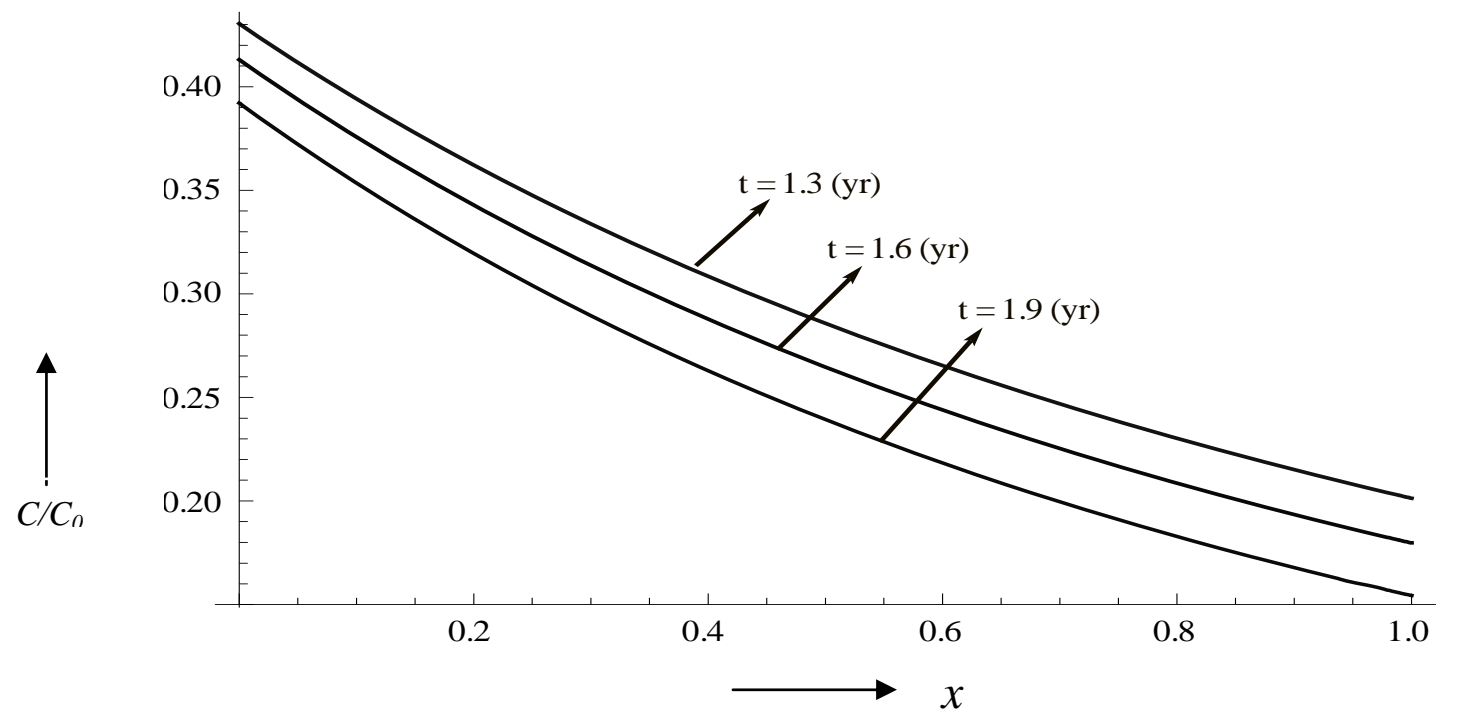

Figure 2b. Distribution behavior of solute in absence of source concentration for spatially dependent dispersion at different time.

It may be observed that in the presence of pollutants source, concentration values decrease with position and increase with time. Once the source of the pollution is removed, the input concentration becomes zero and at a particular position concentration level starts decreasing with time, and appears to settle at a minimum level. The decrease (in the presence of the source) or increase (in the absence of the source) is faster at the initial stage. Analytical methods basically provide solutions to governing equations of groundwater flow and contaminant transport with simplified boundary conditions and hydro-geological and chemical properties and are useful to predicting the more comprehensive numerical solutions. But it is extremely difficult or even impossible to derive analytical solutions to solute transport when a more complex system is considered, e.g., a system with one or more aspects among moving and periodic boundary conditions, variable-density flow, heterogeneity of geological settings, physical, chemical, and biological degradations, and multiple dimensions.

\section{Conclusion}

In the present work, the advection-dispersion equation in one-dimension are solved analytically for varying pulse type input source in semi-infinite domain for time dependent dispersion along uniform flow and spatially dependent solute dispersion along non-uniform flow. It has been possible with the introduction of a new transformation in independent variable. Solute transport due to dispersion and advection, originating from a point stationary source of pulse type of varying nature through a homogeneous/heterogeneous semi-infinite horizontal medium, is studied. The heterogeneity is described by position dependent linear non-homogeneous expression for the velocity. The variation in dispersion parameter due to heterogeneity is considered proportional to square of that in the velocity.

\section{Acknowledgement}

This work is part of the Post Doctoral Fellowship program. Financial assistance provided in the form of UGC-Dr. D. S. Kothari Post Doctoral Fellowship, University Grant Commission, Government of India, to both the authors are gratefully acknowledged.

\section{References}

Al-Niami, A. N. S., and Rushton, K. R.. 1977. Analysis of flow against dispersion in porous media. Journal of Hydrology, Vol. 33, pp. 87-97.

Banks, R.B., and Ali, J. 1964. Dispersion and adsorption in porous media flow. Journal of Hydraulic Division, Vol. 90, pp. 13-31.

Banks, R.B., and Jerasate, S.R. 1962. Dispersion in unsteady porous media flow, Journal of Hydraulic Division, Vol. 88, pp. 1-21.

Bastian, W.C., and Lapidus, L. 1956. Longitudinal diffusion in ion exchange and chromatographic columns. Journal of Physical Chemistry, Vol. 60, pp. 816-817.

Chen, J.-S., Liu, C.W., Hsu, H.T. and Liao, C.M.. 2003. A laplace transform power series solution for solute transport in a 
convergent flow field with scale-dependent dispersion. Water Resources Research, Vol. 39, No. 8, pp. 1229.

Crank, J.. The mathematics of diffusion, Oxford University Press, London, 1975.

Crank, J.. Free and moving boundary problems, Clarendon Press, Oxford University, London, 1984.

Hunt, B. 1978. Dispersion calculations in non-uniform seepage. Journal of Hydrology, Vol. 36, pp. 261-277.

Jaiswal, D.K., Kumar, A., Kumar, N. and Yadav, R.R.. 2009. Analytical solutions for temporally and spatially dependent solute dispersion of pulse type input concentration in one dimensional semi-infinite media. Journal of Hydro-environment Research, Vol. 2, pp. 254-263.

Kartha, S. A. and Srivastava, R. 2008. Effect of immobile water content on contaminant transport in unsaturated zone, Journal of Hydro-environment Research, Vol. 1, pp. 206-215.

Kumar, N. 1983. Unsteady flow against dispersion in finite porous media. Journal of Hydrology, Vol. 63, pp. 345-358.

Kumar, A., Jaiswal, D.K. and Kumar, N.. 2010. Analytical solutions to one-dimensional advection-diffusion with variable coefficients in semi-infinite media. Journal of Hydrology, Vol. 380, pp. 330-337.

Lindstrom, F.T., and Boersma, L.. 1989. Analytical solutions for convective dispersive transport in confined aquifers with different initial and boundary conditions. Water Resources Research, Vol. 25, pp. 241-256.

Marino, M.A. 1974. Distribution of contaminants in porous media flow. Water Resources Research, Vol. 10, pp. 1013-1018.

Meerschaert, Mark M. and Tadjeran, Charles 2004. Finite difference approximations for fractional advection-dispersion flow equations, Journal of Computational and Applied Mathematics, Vol. 172, pp. 65-77.

Mohebbi, A. and Dehghan, M. 2010. High-order compact solution of the one-dimensional heat and advection-diffusion equations, Applied Mathematical Modelling, Vol. 34, pp. 3071-3084.

Moreira, D. M., Vilhena, M. T. Tirabassi, T., Costa, C. and Bodmann, B. 2006. Simulation of pollutant dispersion in the atmosphere by the laplace transform: the ADMM approach, Water, Air, and Soil Pollution, Vol. 177, pp. 411-439.

Ogata, A. 1970. Theory of dispersion in granular media. U S Geo. l Sur. Prof. Paper, 411-I, 34.

Su, Ninghu, Sander, G.C., Liu, Fawang, Anh, Vo and Barry, D.A. 2005. Similarity solutions for solute transport in fractal porous media using a time and scale-dependent dispersivity, Applied Mathematical Modelling, Vol. 29, pp. 852-870.

Sirin, H. 2006. Ground water contaminant transport by non divergence-free, unsteady and non-stationary velocity fields. Journal of Hydrology, Vol. 330, pp. 564-572.

Smedt, F.D. 2006. Analytical solutions for transport of decaying solutes in rivers with transient storage. Journal of Hydrology, Vol. 330, pp. 672-680.

van Kooten, J.J.A. 1996. A method to solve the advection-dispersion equation with a kinetic adsorption isotherm. Advances in Water Resources, Vol. 19, pp. 193- 206.

Warrick, A.W., Kichen, J. H. and Thames, J. L.. 1972. Solutions for miscible displacement of soil water with time-dependent velocity and dispersion coefficients. Soil Sci Soc Am J., Vol. 36, pp. 863-867.

Yates, S.R. 1990. An analytical solution for one-dimensional transport in heterogeneous porous media, Water Resources Research, Vol. 26, pp. 2331-2338.

Zoppou, C., and Knight, J.H.. 1997. Analytical solutions for advection and advection-diffusion equation with spatially variable coefficients. Journal of Hydraulic Engineering, Vol. 123, pp. 144-148.

\section{Biographical notes}

Dr. Dilip Kumar Jaiswal received Ph.D. from Banaras Hindu University, Varanasi, India in 2009. He is a Post Doctoral Fellow in the Department of Mathematics \& Astronomy, Lucknow University, Lucknow, India. He has published/accepted more than ten research papers in international Journals.

Atul Kumar is a Post Doctoral Fellow in the Department of Mathematics \& Astronomy, Lucknow University, Lucknow, India. He has published/accepted more than five research papers in international Journals.

Received October 2010

Accepted January 2011

Final acceptance in revised form January 2011 